\title{
Shot noise in diffusive conductors: A quantitative analysis of electron-phonon interaction effects
}

\author{
Y. Naveh, D. V. Averin, and K. K. Likharev \\ Department of Physics and Astronomy, State University \\ of New York, Stony Brook, NY 11794-3800
}

(May 17, 2018)

\begin{abstract}
Using the 'drift-diffusion-Langevin' equation, we have quantitatively analyzed the effects of electron energy relaxation via their interaction with phonons, generally in presence of electron-electron interaction, on shot noise in diffusive conductors. We have found that the noise power $S_{I}(\omega)$ (both at low and high observation frequencies $\omega$ ) drops to half of its 'mesoscopic' value only at $\beta \gtrsim 100$, where $\beta$ is the ratio of the sample length $L$ to the energy relaxation length $l_{\mathrm{ph}}$ (the latter may be much larger then the dephasing length). It means in particular that at low temperatures the shot noise may be substantial even when $L \sim 10^{-2}-10^{-1} \mathrm{~cm}$, and the conductor is 'macroscopic' in any other respect.
\end{abstract}

Macroscopic metallic conductors, in which electrons are in local equilibrium, do not exhibit non-equilibrium ("shot") noise at low voltages. On the other hand, short diffusive conductors (with length $L$ much shorter than the effective electron-phonon relaxation length $l_{\mathrm{ph}}=$ $\left.\sqrt{D \tau_{\mathrm{ph}}}\right)$ do show this effect as soon as the average energy $\mathrm{eV} / 2$ acquired from the electric field becomes larger than the energy scale $T$ of equilibrium thermodynamic fluctuations 1 - Electron-electron scattering (which become important at $L>l_{e e}=\sqrt{D \tau_{e e}}$ ) affect the shot noise intensity only slightly at low frequencies 4 , 5 , though at frequencies above the reciprocal Thouless time $\tau_{T}^{-1}=D / L^{2}$ their effect is more substantialla. This low sensitivity of shot noise to electron-electron scattering, as well as to screening 3 , may be readily understood: electron-electron interaction cannot drain the energy supplied by the external electric field from the electron subsystem, which is thereby "overheated". Shot noise may be considered as a direct result of this deviation from equilibrium.

The effect of electron-phonon interaction is quite different: it may drain extra energy from the electron subsystem, bring it closer to local thermal equilibrium, and hence suppress the shot noise. The analysis of these effects has heen addressed in many recent works, both analytically 2. 10 and using numerical Monte-Carlo simulations11 (see also Ref.12). While describing the reduction of shot noise with growing values of $\beta \equiv L / l_{\mathrm{ph}}$, these works cannot provide realistic values for the noise at $\beta \gtrsim 1$. For example, assuming a simplifying form of the electron-phonon interaction, it was concluded in Ref.G that the low-frequency noise behaves as $S_{I} \sim e^{-\beta^{2}}$. On the other hand, a crude partition of the sample into $L / l_{\mathrm{ph}}$ segments 10,10 , or, alternatively, the assumption of a constant relaxation time $\mathrm{B}$, lead to the conclusion that the noise should vanish much slower, as $\beta^{-1}$, since such approaches neglect the dependence of the relaxation rate on the excitation enerory. This dependence was taken into account by Nagaev2, B. By asymptotically solving the Boltzmann equation, he showed that for the case of zero temperature and zero frequency, and in the limit $\beta \gg 1$, the noise intensity behaves as $S_{I} \sim \beta^{-2 / 5}$. However, the full dependence of the noise on $\beta$, as well as the more realistic case of finite temperatures and frequencies, has not yet been studied. Such a study was the goal of this work We have used the 'drift-diffusion-Langevin' equation 3 , 6 to calculate the current noise power as a function of the ratio $\beta$, the temperature $T$, and $\omega$, for the two limiting values of the ratio $\gamma=L / l_{e e}$.

The drift-diffusion-Langevin equation 3 , 3 is based on integration of the Boltzmann-Langevin equation 13 over the electron energies. Its self-consistent solution with the Poisson equation that accounts for screening in the system enables one to calculate noise power at arbitrary frequencies 3 . The outcome of this approach may be summarized as the following simple recipe: for a conductor with uniform cross-section, the spectral density of current fluctuations at any point $x$ (along conductor's length) is

$$
S_{I}(x ; \omega, T)=\frac{2 A}{L^{2}} \int_{-\frac{L}{2}}^{\frac{L}{2}}\left|K\left(x, x^{\prime} ; \omega\right)\right|^{2} \mathcal{S}\left(x^{\prime} ; T\right) d x^{\prime}
$$

with $A$ the cross-sectional area. The local noise correlator $\mathcal{S}(x ; T)$ is given by

$$
\mathcal{S}(x ; T)=2 \sigma(x) \int_{0}^{\infty} d E f_{s}(E, x ; T)\left[1-f_{s}(E, x ; T)\right]
$$

with $\sigma(x)$ the local conductivity and $f_{s}(E, x ; T)$ the momentum-symmetric part of the local (steady state) distribution function at a total energy $E$. The response function $K\left(x, x^{\prime} ; \omega\right)$ equals 1 at zero frequency, but at finite frequencies it is dependent on the specific geometry of the conductor and its electrodynamic environment, always obeying the following sum rule:

$$
\frac{1}{L} \int_{-\frac{L}{2}}^{\frac{L}{2}} K\left(x, x^{\prime} ; \omega\right) d x^{\prime}=1
$$

In this work, we concentrate on the geometry which looks most promising for experimental observation of the considered effects, namely, a thin and long conductor close to a ground plane. For the quantitative applicability of our results, thickness of the conductor and its 
distance from the ground plane should be much smaller than $L$, but its second transversal dimension (parallel to the ground plane) may be arbitrary. (As a result the model is applicable, e.g., to a two dimensional electron gas gated by a close electrode, which also serves as the ground plane). In this geometry the response function for noise current in the external electrodes is very simple 6 :

$$
K^{e}\left(x^{\prime} ; \omega\right)=\kappa \frac{L}{2} \frac{\cosh \left(\kappa x^{\prime}\right)}{\sinh (\kappa L / 2)} .
$$

Here $\kappa=\sqrt{-i \omega / D^{\prime}}, D^{\prime}=D+\sigma A / C_{0}, C_{0}$ is the (dimensionless) linear capacitance between the conductor and the ground plane, and $D$ is the diffusion coefficient. The non-equilibrium distribution function $f_{s}(E, x)$ should be found by solving the stationary Boltzmann equation. At this stage it is convenient to use dimensionless quantities $\xi=x / L, \varepsilon=E / \mathrm{eV}$, and $t=T / \mathrm{eV}$. In the diffusion limit $(l \ll L)$, the Boltzmann equation is

$$
-\left(D / L^{2}\right) \frac{d^{2} f_{s}(\varepsilon, \xi)}{d \xi^{2}}=I(\varepsilon, \xi)
$$

with $I(\varepsilon, \xi)$ the collision integral.

We study here two limiting cases. In the first limit, electron-electron scattering within the conductor is negligible, $\gamma=L / l_{e e} \ll 1$, so the collision integral involves only scattering by phonons. For the feformation potential scattering it may be presented as 14

$$
\begin{aligned}
I(\varepsilon, \xi)= & \frac{1}{\tau_{V}} \int_{0}^{\infty} d \omega \omega^{2}\left\{\left(1-f_{s}\right)\left[f_{s}^{+}(1+N)+f_{s}^{-} N\right]\right. \\
& \left.-f_{s}\left[\left(1-f_{s}^{-}\right)(1+N)+\left(1-f_{s}^{+}\right) N\right]\right\}
\end{aligned}
$$

with $f_{s} \equiv f_{s}(\varepsilon, \xi), f_{s}^{ \pm} \equiv f_{s}(\varepsilon \pm \omega, \xi), N \equiv N(\omega) \equiv$ $1 /[\exp (\omega / t)-1]$, and

$$
\frac{1}{\tau_{V}}=\frac{1}{2 \pi}\left(\frac{e V}{2 \hbar k_{F} v_{s}}\right)^{3} \frac{m k_{F}^{2} \Xi^{2}}{\hbar^{2} \rho v_{s}},
$$

with $k_{F}$ the Fermi wavenumber, $m$ the effective mass, $\Xi$ the deformation potential constant, $v_{s}$ the sound velocity, and $\rho$ the mass density of the material. Since the voltage drops only across the conductor, the distribution function must approach the equilibrium distribution at the conductor-electrode interfaces. The boundary conditions for Eq. (5) are therefore

$$
f_{s}(\varepsilon, \mp 1 / 2)=f_{0}(\varepsilon \mp 1 / 2) \equiv \frac{1}{1+\exp \left(\frac{\varepsilon \mp 1 / 2}{t}\right)} .
$$

In the second limit, electron-electron scattering is strong, $\gamma \gg 1$, so the electrons are locallithermalized. The distribution function is then given by 15

$$
f_{s}(\varepsilon, \xi)=\frac{1}{1+\exp \left[\frac{\varepsilon+\xi}{t_{e}(x)}\right]} .
$$

The equation for the electron temperature $t_{e}(x)=$ $T_{e}(x) / \mathrm{eV}$ in this limit was obtained in Ref. 6 by multiplying the Boltzmann equation by $\varepsilon$ and integrating it with respect to energy. The resulting equation reads

$$
\frac{\pi^{2}}{6} \frac{d^{2}\left[t_{e}^{2}(\xi)\right]}{d \xi^{2}}=24 \zeta(5) \frac{L^{2}}{\tau_{V} D}\left(t_{e}^{5}(\xi)-t^{5}\right)-1,
$$

where $\zeta(5) \simeq 1.04$ is the Riemann zeta function.

$\tau_{V}$ which appears in equations (6, 10$)$ is the energy relaxation time of electrons with typical energy $\mathrm{eV}$. The relaxation length is then $l_{\mathrm{ph}}=\sqrt{D \tau_{V}}$. One can immediately notice from equations $(5 \sqrt{6})$ and $(8-10)$ that the dependence of $f_{s}(E, x)$ on the physical variables of the problem $\mathrm{eV}, T, L$, and $l_{\mathrm{ph}}$ in each of our cases is oply through the parameters $t=T / \mathrm{eV}$ and $\beta=L / l_{\mathrm{ph}} 1 \mathrm{E}$. From equations (1,2, 2, (1) it is then seen that for a uniform conductor $[\sigma(x)=\sigma]$ the only additional parameter which affects the normalized noise value $\alpha=S_{I} / 2 e I$ is $|\kappa| L=\sqrt{\omega \tau_{T}}$, with $\tau_{T}=L^{2} / D^{\prime}$ the effective Thouless time. In particular, at fixed $\omega \tau_{T}$ the only dependence of the noise on the sample length is due to its ratio $\beta$ with the thermalization length.

Equation (5) can be solved analytically in three limiting cases: $\beta=\gamma=0$ (no energy relaxation), $\beta=0, \gamma \rightarrow$ $\infty$ (strong local thermalization), and $\beta \rightarrow \infty$ (strong thermalization to lattice temperature). The corresponding distribution functions are

$$
\begin{aligned}
& f_{s}(\varepsilon, \xi)=(1 / 2+\xi) f_{0}(\varepsilon+1 / 2) \\
& +(1 / 2-\xi) f_{0}(\varepsilon-1 / 2) \quad \beta=\gamma=0, \\
& f_{s}(\varepsilon, \xi)=\left\{1+\exp \left[\frac{\varepsilon+\xi}{t_{h}(\xi)}\right]\right\}^{-1} \quad \beta=0, \gamma \rightarrow \infty \text {, } \\
& f_{s}(\varepsilon, \xi)=\left[1+\exp \left(\frac{\varepsilon+\xi}{t}\right)\right]^{-1} \quad \beta \rightarrow \infty,
\end{aligned}
$$

with the hot-electron temperature $t_{h}(\xi)=$ $\sqrt{t^{2}+3\left(1-4 \xi^{2}\right) / 4 \pi^{2}}$. The frequency and temperature dependences of the noise power in these limiting cases were given in 6 .

In order to study the crossover region (i.e., at finite values of $\beta$ ) we solve equations (5) and (10) numerically. Results for the distribution functions for several values of $\beta$ are presented in figures 1 and 2 . Figure 1 shows the dependence of $f_{s}(\varepsilon, \xi)$ on energy and position at $t=0.01$, with no electron-electron scattering. Plots (a) and (f) correspond to the limiting cases described by Eqs. (11a) and (11), respectively. At large $\beta$ the integral in Eq. (6) must be small, and so the distribution is close to a Fermi Dirac distribution in most of the sample. However, the effective temperature of this distribution approaches the lattice temperature $t=0.01$ only at $\beta \gtrsim 10^{5}$. This form of the distribution function means that a perturbation expansion of Eq. (5) around the distribution at $\beta \rightarrow \infty$ gives results which are far from reality for values of $\beta$ smaller than $\sim 10^{5}$. Note also that at distances of the 
order $L / \beta^{1 / 2}$ from the edges of the sample the distribution is much sharper than in the bulk. We will show that this fact has strong implications on the high frequency noise.
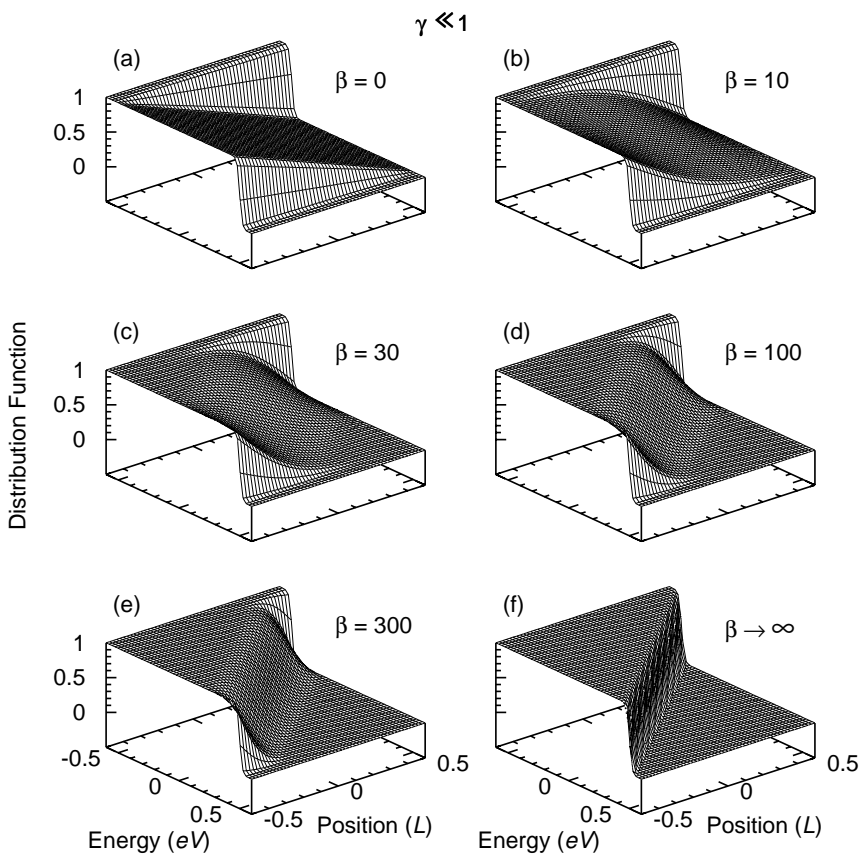

FIG. 1. The symmetric part of the electron distribution function $f_{s}(\varepsilon, \xi)$ for different values of $\beta=L / l_{\mathrm{ph}}$ and for weak electron-electron interaction $\left(\gamma=L / l_{e e} \ll 1\right)$. For all curves, $T=0.01 \mathrm{eV}$.


FIG. 2. (a) Same as in Fig. 1 but for the case $\gamma \gg 1$. (b) The dependence of the electron temperature $t_{e}$ on the position and on the electron-phonon interaction parameter $\beta$.
Figure 2(a) shows the distribution function for the case of strong electron-electron scattering, and $\beta=10$. At any other $\beta$, the plot looks generally the same, with the only difference being that the width of the distribution on the diagonal $\varepsilon+\xi=0$ would change according to the local electron temperature $t_{e}(\xi ; \beta)$. The dependence of this temperature on position and on $\beta$ is shown in Fig 2(b).

Figure 3 shows our main result: the normalized spectral density as a function of $\beta$ for various frequencies. At $\omega \tau_{T}, t \ll 1$ and $\beta \gg 1$, Nagaev's asymptotic result. 2 . 4 are reproduced; in our notation they read

$$
\begin{aligned}
& S_{I} / 2 e I \simeq \frac{1.2}{\beta^{2 / 5}} \quad(\gamma \ll 1), \\
& S_{I} / 2 e I \simeq \frac{1.05}{\beta^{2 / 5}} \quad(\gamma \gg 1) .
\end{aligned}
$$

However, for the case $\gamma \ll 1$ the result (12a) relies on the fact that at large $\beta$ the electron distribution function is invariant to diagonal transformations $\xi \rightarrow \xi+\xi_{0}, \varepsilon \rightarrow$ $\varepsilon-\xi_{0}$. As mentioned above [cf. Fig. 1(e)], this invariance is not valid at distances $\xi \sim \beta^{-1 / 2}$ from the edges of the sample. Due to the form of the response function (4) the high frequency noise is sensitive to the distribution of electrons only at distances $\xi \sim 1 /|\kappa L|=\left(\omega \tau_{T}\right)^{-1 / 2}$ from the edges. Therefore, the noise behaves with its asymptotic power-law form only at $\beta \gg \omega \tau_{T}$, as clearly seen in Fig. 3 (solid lines).

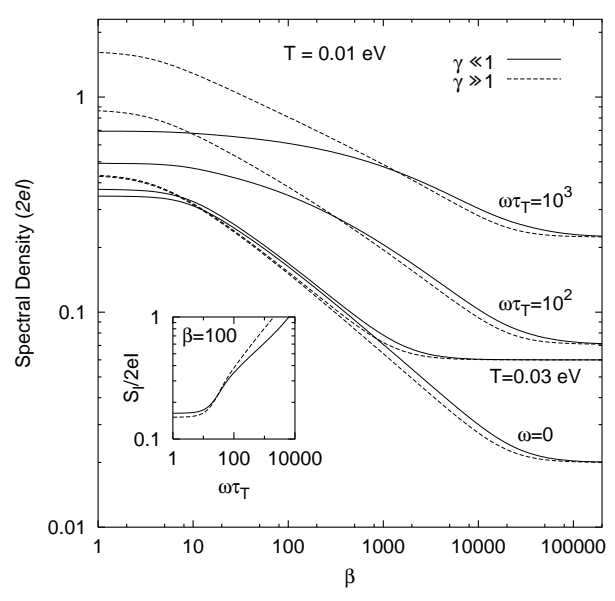

FIG. 3. The dependence of the noise spectral density on $\beta$ for $t \equiv T / e V=0.01$ at various frequencies, for the two cases: $L \ll l_{e e}$ (solid lines) and $L \gg l_{e e}$ (dashed lines). The same dependence for $t=0.03$ and zero frequency is also shown. Inset: the dependence of the spectral density on frequency at $t=0.01$ and $\beta=100$.

A similar analysis can be done for the case $\gamma \gg 1$ (dashed lines in Fig. 3). Here $S_{I} \sim \beta^{-2 / 5}$ because $f_{s}(\varepsilon, \xi)$ does not depend on $\xi$ at $\beta \gg 1$. As can be deduced from Eq. (10), this constancy of $f_{s}$ does not hold at distances smaller than $\xi \sim \beta^{-2 / 5}$ from the boundaries, and so, by 
the same arguments as for $\gamma \ll 1$, the high frequency noise behaves as Eq. (12b) only at $\beta \gg\left(\omega \tau_{T}\right)^{5 / 4}$. In contrast to the case of $\gamma \ll 1$, however, in this case the noise at $1 \ll \beta<\left(\omega \tau_{T}\right)^{5 / 4}$ does not retain its $\beta=0$ value, because the electron temperature decreases with $\beta$ also near the boundary, see Fig. 2(b). The inset of Fig. 3 shows the frequency-dependence of the noise spectral density at $\beta=100$ and $t=0.01$. More details of the dependence of the noise on frequency and temperature at $\beta=0$ were given elsewhere 6 .

To summarize, we have performed numerical calculations of the non-equilibrium noise in samples with strong elastic scattering and with an arbitrary strength of electron-acoustic phonon scattering. We have shown that the shot noise decreases very slowly with the sample length, and is of the order of the Schottky value even for $L / l_{\mathrm{ph}} \sim 100$. In experiment, it means that when dealing with otherwise 'macroscopic' samples (at low temperatures, of the order of one millimeter), the a priori assumption of vanishing shot noise may be wrong. In view of the persistent improvements in accuracy of noise measurements in recent years 1721 and the growing general interest in the relations between inelastic relaxation, fluctuations, and dephasing22, we believe that there is a considerable chance of experimental confirmation of this prediction.

The work was supported in part by DOE's Grant \#DE-FG02-95ER14575.

${ }^{1}$ C.W.J. Beenakker and M. Büttiker, Phys. Rev. B 46, 1889 (1992).

${ }^{2}$ K.E. Nagaev, Phys. Lett. A 169, 103 (1992).

${ }^{3}$ Y. Naveh, D.V. Averin, and K.K. Likharev, Phys. Rev. Lett. 79, 3482 (1997).

${ }^{4}$ K. E. Nagaev, Phys. Rev. B 32, 4740 (1995).

${ }^{5}$ V. I. Kozub and A. M. Rudin, Surf. Sci. 361/362, 722 (1996).

${ }^{6}$ Y. Naveh, D.V. Averin, and K.K. Likharev, condmat/9801188; submitted to Phys. Rev. B.

${ }^{7}$ A. Shimizu and M. Ueda, Phys. Rev. Lett. 69, 1403 (1992);
M. Ueda and A. Shimizu, J. Phys. Soc. Japan, 62, 2994 (1993).

${ }^{8}$ R. Landauer, Phys. Rev. B 47, 16427 (1993).

${ }^{9}$ R. C. Liu and Y. Yamamoto, Phys. Rev. B 49, 10520 (1994); Phys. Rev. B 50, 17411 (1994).

10 M. Buttiker, in Noise in Physical Systems and $1 / f$ Fluctuations, Edited by v. Bareikis and R. Katilius (World Scientific, Singapore, 1995).

${ }^{11}$ R. C. Liu, P. Eastman, and Y. Yamamoto, Solid State Comm. 102, 785 (1997). In this work only strictly onedimensional electrons without screening where studied.

12 The effects of phonon relaxation on the noise in ballistic structures where studied in Refs 11 , and in V. L. Gurevich and A. M. Rudin, Pis'ma Zh. Eksp. Teor. Fiz. 62, 13 (1995) [JETP Lett. 62, 13 (1995)]; Phys. Rev. B 53, 10078 (1996).

13 Sh.M. Kogan and A.Ya. Shul'man, Zh. Eksp. Teor. Fiz. 56, 862 (1969) [Sov. Phys. JETP 29, 467 (1969)]; Sh. Kogan, Electronic noise and fluctuations in solids (Cambridge, Cambridge, 1996), p. 80.

${ }^{14}$ V. F. Gantmakher and Y. B. Levinson, Carrier Scattering in Metals and Semiconductors (North-Holland, Amsterdam, 1987).

${ }^{15}$ An experimental verification of this distribution was recently reported by H. Pothier, S. Gueron, N. O. Birge, D. Esteve, and M. H. Devoret, Phys. Rev. Lett. 79, 3490 (1997).

16 This statement is correct only in the regular case when the voltage is smaller than the Debye temperature $\Theta_{D}$. Otherwise, the energy relaxation time does not depend on the voltage 14, and therefore $f_{s}$ depends separately on $V$ and $T$. The case $\Theta_{D} \leq e V$ is not very practical due to the strong heating of the lattice in this case.

${ }^{17}$ F. Liefrink, J. I. Dijkhuis, M. J. M. de Jong, L. W. Molenkamp, and H. van Houten Phys. Rev. B 49, 14066 (1994).

18 M. Reznikov, M. Heiblum, H. Shtrikman, and D. Mahalu, Phys. Rev. Lett. 75, 3340 (1995).

19 A.H. Steinbach, J.M. Martinis, and M.H. Devoret, Phys. Rev. Lett. 76, 3806 (1996).

${ }^{20}$ M. Henny, H. Birk, R. Huber, C. Strunk, A. Bachtold, M. Krüger, and C. Schönenberger, Appl. Phys. Lett. 71, 773 (1997).

${ }^{21}$ R. J. Schoelkopf, P. J. Burke, A. Kozhevnikov, D. E. Prober, and M. J. Rooks, Phys. Rev. Lett. 78, 3370 (1997).

22 See, e.g., P. Mohanty, E. M. Q. Jariwala, and R. A. Webb, Phys. Rev. Lett. 78, 3366 (1997). 